\title{
Ambient connections realising conformal Tractor holonomy
}

\author{
Stuart Armstrong* and Thomas Leistner ${ }^{\dagger}$
}

\begin{abstract}
For a conformal manifold we introduce the notion of an ambient connection, an affine connection on an ambient manifold of the conformal manifold, possibly with torsion, and with conditions relating it to the conformal structure. The purpose of this construction is to realise the normal conformal tractor holonomy as affine holonomy of such a connection. We give an example of an ambient connection for which this is the case, and which is torsion free if we start the construction with a C-space, and in addition Ricci-flat if we start with an Einstein manifold. Thus for a $C$-space this example leads to an ambient metric in the weaker sense of Čap and Gover, and for an Einstein space to a Ricci-flat ambient metric in the sense of Fefferman and Graham.
\end{abstract}

\section{Introduction}

Conformal geometry has been studied for a long time. Cartan's Car23 techniques of 'moving frames' developed into principal bundles and the Cartan connection. The equivalent 'Tractor' connection has been developed by T. Thomas (Tho26] and [Tho32]), further developed by T.N. Bailey, M.G. Eastwood and A.R. Gover (BEG94 and Eas96), and extensively treated in papers of A.R Gover and A. Čap (e.g. ČG02 and ČG03), which being a vector bundle construction, allows for more explicit calculations. One interesting invariant of the Tractor connection is its holonomy group, and the geometric consequences of this group. Classification of these groups is essentially finished for Riemannian signature in Arm05, see also Leit04.

A major technique in this classification was the use of cone-constructions for conformally Einstein manifolds with non-zero Einstein constant, and a degenerate cone construction in the Ricci-flat case [Lei05, which are both instances of the original ambient construction of C. Fefferman and C. R. Graham (FG85], also [FG02] and [FH03). A qualitative description generalising the original ambient construction was given in [CG03, where also the relation to the Tractor bundle is described. This paper seeks to generalise both cone-constructions and the ambient metric construction of [CG03 to generate an affine connection on an ambient manifold which is metric but possibly with torsion and with same holonomy group as the Tractor connection - indeed the tangent bundle of this ambient manifold becomes identified with the Tractor bundle.

In this paper, we will start by introducing the basic concepts of tractor bundles in Section 2. We shall also define associated structures, and set up the notation we shall be using

\footnotetext{
*stuart.armstrong@st-cross.oxford.ac.uk

${ }^{\dagger}$ tleistne@maths.adelaide.edu.au

Date: June 15, 2006

2000 MSC: 53C29; 53A30

Keywords: Holonomy groups; conformal holonomy; ambient construction
} 
throughout. Section 3 defines recalls the notion of an ambient manifold with ambient metric following CG03, introduces the notion of an ambient connection, and demonstrates many of the properties it must have. Section 4 then looks more particularly at the intermesh between an ambient manifold and the Tractor bundle and how the properties of one transfer across to the other.

What is presented in Section 5 was the inspiration for this paper: an example of an ambient connection is given the holonomy of which equals to the normal conformal Tractor holonomy, and that, we hope, could lead to useful results for calculating Tractor holonomy groups. For now it serves two purposes: on the one hand it demonstrates geometrically the algebraic fact proved in Lei05 that the Tractor holonomy algebra of a conformal structure which contains a metric with vanishing Cotton-York tensor is a Berger algebra. On the other hand our construction contains the Einstein cone and Ricci-flat construction as special cases. Finally, in Section 6 we give some useful properties of the holonomy of ambient connections, for future constructions.

Acknowledgements. The authors acknowledge the hospitality at the Erwin Schödinger International Institute for Mathematical Physics (ESI) in Vienna, and would like to thank the organisers of the programme Geometry of Pseudo-Riemannian Manifolds with Applications in Physics at the ESI, during which the discussion on the topic started.

\section{Preliminaries}

Let $M$ be a smooth manifold of dimension $n$.

Definition 2.1 (Weight Bundles). Weight bundles are defined as line bundles that are powers of the top wedge product of the cotangent bundle. In details,

$$
\mathcal{E}[-n]=\wedge^{n} T^{*} M
$$

and

$$
\mathcal{E}[a]=(\mathcal{E}[-n])^{-\frac{a}{n}}
$$

If $\mathcal{B}$ is a vector bundle associated to the tangent bundle, we designate the tensor product $\mathcal{B} \otimes \mathcal{E}[a]$ as $\mathcal{B}[a]$. Weight bundles are a way of formalising the notion of conformal weights in the following sense. If we are given a conformal structure $[g]$ as a equivalence class of metrics with $\mathbb{R}^{+}$action

$$
(t, g) \rightarrow t^{2} g
$$

then the action on the determinant becomes

$$
(t, \operatorname{det}(g)) \rightarrow t^{n} \operatorname{det}(g)
$$

and since $\operatorname{det}(g) \in \Gamma(\mathcal{E}[-n])$, it can be seen that the conformal structure $[g]$ is isomorphic to a non-degenerate section $\hat{g}$ of $\left(\odot^{2} T^{*} M\right)[2]$.

There are many equivalent ways of describing the conformal structure, quite apart from equivalence classes of proportionate metrics, such as classes of conformal connections, a principal $C O(p, q)$-bundle for the tangent bundle, etc. We will use the description in terms of 
the standard Tractor bundle (see Tho26, [BEG94, and [CG02]) equipped with the standard tractor connection. This construction defines the conformal structure entirely; however, for a given conformal structure, the Tractor connection is not unique. Similarly to the LeviCivita connection for metric structures, there is a unique normal Tractor connection for each conformal structure. For our purposes a conformal standard Tractor bundle with Tractor connection and the normal connection amongst them are best described by the following properties, from which the conformal structure can be recovered.

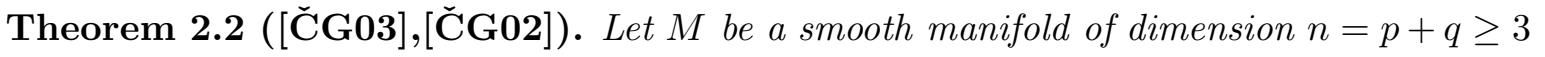
and $\mathcal{T}$ be a vector bundle over $M$ of rank $n+2$.

1. Suppose $\mathcal{T}$ admits a metric $h$ of signature $(p+1, q+1)$, an injective vector bundle homomorphism $\mathcal{E}[-1] \hookrightarrow \mathcal{T}$ with image $\mathcal{T}^{1}$, and a covariant derivative $\vec{\nabla}$ such that

(a) $\mathcal{T}^{1}$ is light-like,

(b) $\vec{\nabla} h=0$, and

(c) the following non-degeneracy condition is satisfied: for any $x \in M$ and any section $\sigma \in \Gamma\left(\mathcal{T}^{1}\right)$, non-zero at $x$, the map $\vec{\nabla} \sigma: T_{x} M \rightarrow \mathcal{T}_{x}$ is non-degenerate.

Then $\left(\mathcal{T}^{1}\right)^{\perp} / \mathcal{T}^{1} \simeq T M[-1]$ and $(\mathcal{T}, h, \vec{\nabla})$ is a standard tractor bundle for the conformal structure defined by the restriction of $h$ onto $\left(\mathcal{T}^{1}\right)^{\perp} / \mathcal{T}^{1}$.

2. A standard conformal Tractor connection $\vec{\nabla}$ on $\mathcal{T}$ is normal if and only if its curvature $\mathcal{R}$ maps $\mathcal{T}^{1}$ onto $\mathcal{T}^{1}$ and the Ricci contraction of $W \in \Lambda^{2} T^{*} M \otimes \operatorname{End}(T M[-1])$, which is defined by $W(X, Y)[Z]=[\mathcal{R}(X, Y) Z]$ using the identification $T M[-1] \simeq\left(\mathcal{T}^{1}\right)^{\perp} / \mathcal{T}^{1}$, vanishes. With these normality conditions $\left(\mathcal{T}, \mathcal{T}^{1}, h, \vec{\nabla}\right)$ is uniquely determined by the conformal structure up to isomorphism.

To see the first point, notice that the conditions imply that $\vec{\nabla} \sigma \in \Gamma\left(T M \otimes\left(\mathcal{T}^{1}\right)^{\perp}\right)$ and that for any choice of $\sigma$, one has a splitting of

$$
\mathcal{T}=\mathcal{E}[1] \oplus T M[-1] \oplus \mathcal{E}[-1]
$$

where $T M[-1]$ is the image of $T M$ under $\vec{\nabla} \sigma$, and $\mathcal{E}[1]$ is the span of a section $\sigma^{\prime}$, with $\sigma^{\prime} \perp T M[-1]$ and $h\left(\sigma^{\prime}, \sigma\right)=-1$. This allows us to project $\vec{\nabla}$ to get a connection $\nabla$ on $T M[-1]$, which conserves $\hat{g}=\left.h\right|_{T M[-1]}$ - in other words, conserves a conformal structure. Since $T M[-1] \oplus E[-1] \cong\left(\mathcal{T}^{1}\right)^{\perp}$, different non-zero choices of $\sigma$ give the same $\hat{g}$ - though different $\nabla$ 's.

Using $\sigma$ to identify $T M[-1]$ with $T M, \nabla$ then descends to a tangent bundle connection, preserving the tangent metric $\sigma^{-2} \hat{g}$. We call these $\nabla$ preferred connections of the Tractor connection $\vec{\nabla}$. The invariance of $\mathcal{T}^{1}$ under the curvature of the Tractor connection then is equivalent with the preferred connections $\nabla$ being torsion-free. This is because, in the previous notation, the $T M[-1] \cong\left(\mathcal{T}^{1}\right)^{\perp} / \mathcal{T}^{1}$ component of $R(X, Y) \sigma$ is just $\nabla_{X} Y-\nabla_{Y} X-$ $[X, Y]$.

So now pick a preferred $\nabla$, which preserves a metric $g$, and (since it is torsion-free) is the Levi-Civita connection of $g$. Given the associated splitting

$$
\mathcal{T}=\mathcal{E}[1] \oplus T M[-1] \oplus \mathcal{E}[-1],
$$


the normal Tractor connection $\vec{\nabla}$ is given as

$$
\vec{\nabla}_{X}=\nabla_{X}+X+\mathrm{P}(X) \text {. }
$$

Here $\mathrm{P}$ is the Schouten tensor of $\nabla$ defined by

$$
\mathrm{P}_{i j}=-\frac{1}{n-2}\left(\operatorname{Ric}_{i j}-\frac{1}{2 n-2} R \hat{g}_{i j}\right)
$$

Ric being the Ricci tensor and $R$ the scalar curvature of $\nabla$. To understand the action of $X$ and $\mathrm{P}(X)$ in (2), let $\nu, A$ and $\sigma$ be sections of $\mathcal{E}[1], T M[-1]$ and $\mathcal{E}[-1]$, respectively. Then the action is

$$
\begin{aligned}
& X \cdot \nu=0 \\
& X \cdot A=\hat{g}(X, A) \in \Gamma(\mathcal{E}[1]) \\
& \mathrm{P}(X) \cdot \nu=\nu \hat{g}^{-1}(\mathrm{P}(X)) \quad \in \Gamma(T M[-1]) \\
& X \cdot \sigma=\sigma X \\
& \in \Gamma(T M[-1]) \\
& \mathrm{P}(X) \cdot A=\mathrm{P}(X)\lrcorner A \quad \in \Gamma(\mathcal{E}[-1]) \\
& \mathrm{P}(X) \cdot \sigma=0 \text {. }
\end{aligned}
$$

\section{Ambient manifolds, ambient metrics, and ambient connec- tions}

Let $(M, c)$ be a a smooth $n$-dimensional manifold $M$ with conformal structure $c$ of signature $(p, q)$. This is equivalent to a reduction of the linear frame bundle $G l(M)$ to the conformal frame bundle $C O(M)$. It can also be characterised by a principle $\mathbb{R}^{+}$-fibre bundle $\pi: \mathcal{Q} \rightarrow M$ defined as the ray sub-bundle in the bundle of metrics of signature $(p, q)$ given by metrics in the conformal class $c$. In fact, via the $\operatorname{det}^{w / n}$-reduction of $G l(M)$ or $C O(M)$ to $\mathcal{Q}$, it can be viewed as the principal bundle to the weight bundles defined above. The action of $\mathbb{R}^{+}$on $\mathcal{Q}$ shall be denoted by $\varphi$ :

$$
\varphi\left(t, g_{x}\right)=t^{2} g_{x}
$$

From [CG03. we recall the following definitions.

Definition 3.1. Let $\pi: \mathcal{Q} \rightarrow M$ be a conformal structure of signature $(p, q)$ over an $n$ dimensional manifold $M$.

1. An $(n+2)$-dimensional manifold $\widetilde{M}$ is called ambient manifold if

(a) there is a free $\mathbb{R}^{+}$-action $\widetilde{\varphi}$ on $M$, and

(b) an embedding $\iota: \mathcal{Q} \rightarrow \widetilde{M}$ which is $\mathbb{R}^{+}$-equivariant, i.e. the diagram

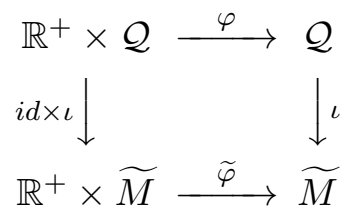

commutes.

2. A metric $h$ on an ambient manifold $\widetilde{M}$ with $\mathbb{R}^{+}$-action $\widetilde{\varphi}$ is called ambient metric, if the following is satisfied: 
(a) If $F$ is the fundamental vector field of $\widetilde{\varphi}$, and $\mathcal{L}$ denotes the Lie derivative, then

$$
\mathcal{L}_{F} h=2 h,
$$

i.e. the metric is homogeneous of degree 2 w.r.t. the $\mathbb{R}^{+}$-action.

(b) For any $g_{x} \in \mathcal{Q}$ the following equality in $\odot^{2} T_{g_{x}}^{*} \mathcal{Q}$ holds:

$$
\left(\iota^{*} h\right)_{g_{x}}=g_{x}(d \pi(.), d \pi(.)) .
$$

We should point out that in the original definition of an ambient metric by C. Fefferman and C.R. Graham in FG85, the additional condition of Ricci-flatness is imposed.

Recall that for $x \in \widetilde{M}$, the fundamental vector field $F(x)$ of the $\mathbb{R}^{+}$-action is defined as the tangent vector of the curve $\widetilde{\varphi}\left(\mathrm{e}^{t}, x\right)$ at $t=0$. Its flow through $x \in \widetilde{M}$ is given by $\varphi_{e^{t}}(x)$. Let us define the 1-form $\phi$ as the dual to the fundamental vector field $F$, i.e. $\phi:=h(F,$.$) .$ Since $F$ is the image under $\iota^{*}$ of the fundamental vector field of the action $\varphi$ on $\mathcal{Q}$, (4) gives that

$$
\iota^{*} \phi=0 \text {. }
$$

This implies that $F$ is of length zero along $\iota(\mathcal{Q})$ and that $h$ is of signature $(p+1, q+1)$. Furthermore it implies that

$$
d \iota^{*} \phi=\iota^{*} d \phi=0 .
$$

Next, we want to consider sections which are homogeneous w.r.t. the $\mathbb{R}^{+}$action on $\widetilde{M}$. We introduce the following notation: If $\mathcal{E}$ is a tensor bundle over the ambient manifold $\widetilde{M}$ or over $\iota(\mathcal{Q})$, then we set for an integer $w$

$$
\Gamma^{w}(\mathcal{E}):=\left\{s \in \Gamma(\mathcal{E}) \mid \mathcal{L}_{F} s=w s\right\}
$$

We write $\Gamma^{w}(\mathbb{R})$ for functions which are homogeneous of degree $w$, i.e. $f \in \Gamma^{w}(\mathbb{R})$ if $\mathcal{L}_{F} f=$ $F(f)=w \cdot f$. Direct calculation using the definition of the Lie derivative and the Jacobi identity for the commutator gives the following properties.

Lemma 3.2. Let $\widetilde{M}$ be a manifold with $\mathbb{R}^{+}$action and corresponding fundamental vector field F. Then it holds:

1. If $\psi \in \Gamma^{w}\left(\otimes^{k} T^{*} \widetilde{M}\right)$, and $X_{i} \in \Gamma^{w_{i}}(T \widetilde{M})$ for $i=1, \ldots, k$ the function $\psi\left(X_{1}, \ldots, X_{k}\right)$ is homogeneous of degree $w+w_{1}+\ldots+w_{k}$.

2. If $X \in \Gamma^{v}(T \widetilde{M})$ and $Y \in \Gamma^{w}(T \widetilde{M})$, then $[X, Y] \in \Gamma^{v+w}(\mathcal{T} \widetilde{M})$.

3. If $f \in \Gamma^{v}(\mathbb{R})$ and $X \in \Gamma^{w}(T \widetilde{M})$, then $X(f) \in \Gamma^{v+w}(\mathbb{R})$.

Of course, the ambient metric defines uniquely the Levi-Civita connection, but for our purposes it is more useful to work with a more general connection which still parallelises the ambient metric. 
Definition 3.3. Let $(\widetilde{M}, h)$ be an ambient manifold with ambient metric $h$ of a conformal structure $\mathcal{Q} \rightarrow M$ in the sense of Definition 3.1. A linear connection $\widetilde{\nabla}$ is called ambient connection if $\widetilde{\nabla} h=0$ and the $(3,0)$-torsion $T^{*}$ of $\widetilde{\nabla}$ satisfies

$$
\mathcal{L}_{F} T^{*}=2 T^{*},
$$

i.e. is homogeneous of degree 2 w.r.t. the $\mathbb{R}^{+}$-action on $\widetilde{M}$.

Recall that the $(2,1)$-torsion of $\widetilde{\nabla}$ is defined as $T_{X, Y}:=\widetilde{\nabla}_{X} Y-\widetilde{\nabla}_{Y} X-[X, Y]$ and the $(3,0)$-torsion as $T^{*}(X, Y, Z):=h\left(T_{X, Y}, Z\right)$. Naturally, (7) is equivalent to $\mathcal{L}_{F} T=0$.

If we define the 1 -form $\phi$ as the dual to the fundamental vector field $F$, i.e. $\phi:=h(F,$.$) ,$ then a direct calculation shows that the covariant derivative of $F$ can be expressed as follows:

$$
h\left(\widetilde{\nabla}_{X} F, Y\right)=h(X, Y)+\frac{1}{2}\left[d \phi(X, Y)-T^{*}(F, X, Y)-T^{*}(F, Y, X)-T^{*}(X, Y, F)\right] .
$$

The homogeneity condition on the torsion implies the following Lemma.

Lemma 3.4. If $X \in \Gamma^{v}(T \widetilde{M})$ and $Y \in \Gamma^{w}(T \widetilde{M})$, then $\widetilde{\nabla}_{X} Y \in \Gamma^{v+w}(T \widetilde{M})$.

Proof. One derives a Koszul formula for a metric connection with torsion:

$$
\begin{aligned}
& 2 h\left(\widetilde{\nabla}_{X} Y, Z\right)=X(h(Y, Z))+Y(h(Z, X))-Z(h(X, Y)) \\
& +h([X, Y], Z)-h([Y, Z], X)+h([Z, X], Y) \\
& +T^{*}(X, Y, Z)-T^{*}(Y, Z, X)+T^{*}(Z, X, Y) \text {. }
\end{aligned}
$$

By the previous lemma the statement follows.

\section{The tractor bundle defined by ambient objects}

In the first part of this section we want to recall the definition of the tractor bundle in terms of an ambient metric due to $\mathrm{CG} 03$. We do no longer distinguish between $\mathcal{Q}$ and the embedded $\iota(\mathcal{Q})$ and between $\varphi$ and $\widetilde{\varphi}$. One considers the restriction on the tangent bundle of an ambient manifold to $\mathcal{Q},\left.T \widetilde{M}\right|_{\mathcal{Q}}$. There is a natural $\mathbb{R}^{+}$-action $\varphi^{*}$ on this bundle defined by

$$
\varphi^{*}\left(t, X_{q}\right):=t^{-1}(d \varphi(t, .))_{q}\left(X_{q}\right) .
$$

The natural projection $\pi:\left.T \widetilde{M}\right|_{\mathcal{Q}} \rightarrow \mathcal{Q}$ is equivariant w.r.t. this action, i.e.

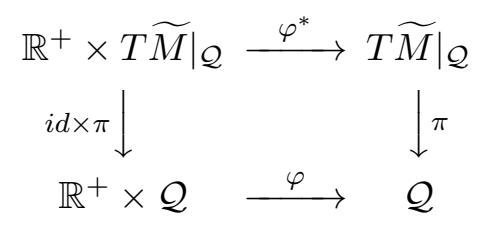

commutes. Therefore the quotient

$$
\mathcal{T}:=\left(\left.T \widetilde{M}\right|_{\mathcal{Q}}\right) / \mathbb{R}^{+}
$$

is a vector bundle over $M \simeq \mathcal{Q} / \mathbb{R}^{+}$of fibre dimension $n+2$. In order to induce a metric and a covariant derivative on $\mathcal{T}$ by the ambient metric and connection we need the identifications of the next lemma. 
Lemma 4.1. There exist the following bijections:

1. $\mathcal{T}_{[g]} \simeq \Gamma^{-1}\left(\left.T \widetilde{M}\right|_{\mathbb{R}^{+} \cdot g}\right)$ for any $[g] \in M=\mathcal{Q} / \mathbb{R}^{+}$and $\mathbb{R}^{+} \cdot g$ the orbit of $g$ under $\varphi$;

2. $\Gamma(\mathcal{T}) \simeq \Gamma^{-1}\left(\left.T \widetilde{M}\right|_{\mathcal{Q}}\right)$;

3. For $M=\mathcal{Q} / \mathbb{R}^{+}$it is $T_{[q]} M \simeq \Gamma^{0}\left(\left.T \mathcal{Q}\right|_{\mathbb{R}^{+} . g}\right) \bmod \mathbb{R} \cdot F$;

4. $\Gamma\left(T\left(\mathcal{Q} / \mathbb{R}^{+}\right)\right) \simeq \Gamma^{0}\left(\left.T \widetilde{M}\right|_{\mathcal{Q}}\right) \bmod \Gamma^{0}(\mathbb{R}) \cdot F$, where $\Gamma^{0}(\mathbb{R})$ denotes functions which are constant along the orbits of $\mathbb{R}^{+}$.

Proof. For $g \in \mathcal{Q}$ we consider the equation

$$
\left(\mathcal{L}_{F} X\right)\left(\varphi_{t}(g)\right)=-X\left(\varphi_{t}(g)\right)
$$

along $\mathbb{R}^{+} \cdot g=\left\{\varphi_{t}(g) \mid t \in \mathbb{R}^{+}\right\}$. This is an ODE which has a unique solution $X\left(\varphi_{t}(g)\right)=$ $\frac{1}{t} d \varphi_{t}\left(X_{g}\right)$ for each initial value $X_{g} \in T_{g} \widetilde{M}$. Recalling that the equivalence relation in $\mathcal{T}$ is the following $X_{\varphi_{t}(g)}=\frac{1}{t} d \varphi_{t}\left(X_{g}\right)$ ensures that there is only one representative lying in $T_{g} \widetilde{M}$ and on the other hand that the solutions obtained by starting at different points in $\mathbb{R}^{+} \cdot g$ are the same. This proves the first point, with the second immediately following. The third point is obtained in the same manner, and implies the last one.

This gives the following conclusions.

Lemma 4.2. The ambient metric defines a metric on the bundle $\mathcal{T}$.

Proof. For $X \in \mathcal{T}_{[g]}$ we denote by $\widetilde{X}$ the corresponding element in $\Gamma^{-1}\left(\left.T \widetilde{M}\right|_{\mathbb{R}^{+} \cdot g}\right)$. Lemma 3.2 ensures that $h(X, Y)$ is constant along the orbits of $\mathbb{R}^{+}$. Hence, by

$$
h_{[g]}^{\mathcal{T}}(\widetilde{X}, \widetilde{Y}):=h_{g}(\widetilde{X}, \widetilde{Y})
$$

a metric on $\mathcal{T}$ is defined.

Proposition 4.3. If $\left.d \phi\right|_{\mathcal{Q}}=0$ and $\left.T^{*}\right|_{\mathcal{Q}}=0$ the ambient connection defines a connection $\nabla^{\mathcal{T}}$ on $\mathcal{T}$ which parallelises $h^{\mathcal{T}}$ and $\left(\mathcal{T}, h^{\mathcal{T}}, \nabla^{\mathcal{T}}\right)$ is a conformal standard tractor bundle for the conformal structure given by $[g]$.

Proof. By assuming $\left.d \phi\right|_{\mathcal{Q}}=0$ and $\left.T^{*}\right|_{\mathcal{Q}}=0$ equation (8) implies that $\widetilde{\nabla}_{X} F=X$. For $X \in \Gamma^{-1}\left(\left.T \widetilde{M}\right|_{\mathcal{Q}}\right)$ this gives on $\mathcal{Q}$ that

$$
0=\widetilde{\nabla}_{F} X-\widetilde{\nabla}_{X} F-[F, X]=\widetilde{\nabla}_{F} X
$$

since $\left.T^{*}\right|_{\mathcal{Q}}=0$. Furthermore Lemma 3.4 ensures that $\widetilde{\nabla}_{X}$ sends $\Gamma^{-1}\left(\left.T \widetilde{M}\right|_{\mathcal{Q}}\right)$ to itself if $X \in \Gamma^{0}\left(\left.T \widetilde{M}\right|_{\mathcal{Q}}\right)$. Denoting again by a tilde the corresponding identifications of 2 . and 4 . in Lemma 4.1 we define the connection on $\mathcal{T}$ by

$$
\widetilde{\nabla_{X}^{\mathcal{T} Y}}:=\widetilde{\nabla}_{\widetilde{X}} \widetilde{Y}
$$

for $X \in \Gamma(T M)$ and $Y \in \Gamma(\mathcal{T})$. By definition this connection is metric w.r.t. $h^{\mathcal{T}}$.

The injection $\mathcal{E}[-1] \hookrightarrow \mathcal{T}$ is given by sending a function $f$ to $[f \cdot F] \in \mathcal{T}$ defining the onedimensional, light-like subbundle $\mathcal{T}^{1}$. The non-degeneracy condition of Theorem 2.2 follows immediately since $\widetilde{\nabla}_{X}(f F)=X(f) F+f \widetilde{\nabla}_{X} F=X(f) F+f X$ along $\mathcal{Q}$. 
Lemma 4.4. Let $\widetilde{M}$ be an ambient manifold with ambient metric $h$ and ambient connection $\widetilde{\nabla}$ such that $\left.d \phi\right|_{\mathcal{Q}}=0$ and $\left.T^{*}\right|_{\mathcal{Q}}=0$. Then the the fundamental vector field $F$ and the curvature $\widetilde{\mathcal{R}}$ of $\widetilde{\nabla}$ satisfy:

1. $\widetilde{\mathcal{R}}(X, Y) F=0$ for all $X, Y \in T \mathcal{Q}$.

2. If in addition $F\lrcorner T=T(., ., F)=0$, then

(a) $h(\widetilde{\mathcal{R}}(F, Z) X, Y)=h(\widetilde{\mathcal{R}}(X, Y) F, Z)$ along $\mathcal{Q}$ for all $X, Y,\left.Z \in T \widetilde{M}\right|_{\mathcal{Q}}$, and

(b) $h(\widetilde{\mathcal{R}}(X, Y) F, Z)=0$ along $\mathcal{Q}$ for all $Z \in T \mathcal{Q}$, and $X,\left.Y \in T \widetilde{M}\right|_{\mathcal{Q}}$.

Proof. A direct calculation based on (8) gives for the ambient curvature $\widetilde{\mathcal{R}}$ of $F$ that

$$
\begin{aligned}
h(\widetilde{\mathcal{R}}(X, Y) F, Z)= & \left(\Lambda_{(1,2)} \widetilde{\nabla} d \phi\right)(X, Y, Z) \\
& +2\left(\Lambda_{(1,2)(3,4)} \widetilde{\nabla} T^{*}\right)(X, Y, F, Z)+\left(\Lambda_{(1,4)} \tilde{\nabla} T^{*}\right)(X, F, Z, Y) \\
& + \text { contractions and symmetrisations of } d \phi \text { and } T^{*},
\end{aligned}
$$

in which $\Lambda_{(i, j)}$ denotes the skew symmetrisation w.r.t. the $(i, j)$-th component. Since it was assumed that $\left.d \phi\right|_{\mathcal{Q}}=0$ and $\left.T^{*}\right|_{\mathcal{Q}}=0$ this implies that

$$
h(\widetilde{\mathcal{R}}(X, Y) F, Z)=0 \text { if } X, Y \in T \mathcal{Q} \text { and }\left.Z \in T \widetilde{M}\right|_{\mathcal{Q}} .
$$

This implies that $\widetilde{\mathcal{R}}(X, Y) F=0$ if $X, Y \in T \mathcal{Q}$.

In order to prove (2) first we notice that for $X, Y,\left.Z \in T \widetilde{M}\right|_{\mathcal{Q}}$ the assumption $\left.F\right\lrcorner T$ implies

$$
\left(\nabla_{X} T\right)(F, Y)=\widetilde{\nabla}_{X}(T(F, Y))=0
$$

and $T(X, Y) \in F^{\perp}$ implies

$$
\nabla_{X} T^{*}(Y, Z, F)=X\left(T^{*}(Y, Z, F)\right)=0
$$

along $\mathcal{Q}$. Since our connection has torsion, the first Bianchi identity does not hold and we do not have in general that $h(\widetilde{\mathcal{R}}(X, Y) F, Z)=h(\widetilde{\mathcal{R}}(F, Z) X, Y)$. But in our situation along $\mathcal{Q}$ this equality holds: for a connection with torsion one gets

$$
\begin{aligned}
\widetilde{\mathcal{R}}(X, Y) Z+\widetilde{\mathcal{R}}(Y, Z) X+\widetilde{\mathcal{R}}(Z, X) Y & = \\
-\left(\widetilde{\nabla}_{X} T\right)(Y, Z)- & \left(\widetilde{\nabla}_{Y} T\right)(Z, X)-\left(\widetilde{\nabla}_{Z} T\right)(X, Y) \\
& +\underbrace{T(X, T(Y, Z))+T(Y, T(Z, X))+T(Z, T(X, Y))}_{=0 \text { along } \mathcal{Q} .}
\end{aligned}
$$

This implies along $\mathcal{Q}$ for $X, Y,\left.Z \in T \widetilde{M}\right|_{\mathcal{Q}}$ and $F$ the fundamental vector field

$$
\begin{aligned}
& \widetilde{\mathcal{R}}(F, X) Y+\widetilde{\mathcal{R}}(X, Y) F+\widetilde{\mathcal{R}}(Y, F) X \\
& \quad=-\widetilde{\nabla}_{F}(T(X, Y))-\widetilde{\nabla}_{X}(T(Y, F))-\widetilde{\nabla}_{Y}(T(F, X))=0
\end{aligned}
$$

and

$$
\begin{aligned}
h(\widetilde{\mathcal{R}}(X, Y) Z+\widetilde{\mathcal{R}}(Y, Z) X & +\widetilde{\mathcal{R}}(Z, X) Y, F) \\
& =-X\left(T^{*}(Y, Z, F)\right)-Y\left(T^{*}(Z, X, F)\right)-Z\left(T^{*}(X, Y, F)\right)=0 .
\end{aligned}
$$


Then, analogously to the torsion free case, one proves that $h(\widetilde{\mathcal{R}}(F, Z) X, Y)=h(\widetilde{\mathcal{R}}(X, Y) F, Z)$ along $\mathcal{Q}$ which is (a).

For (b) we use that $d d \phi=0$ implies that

$$
\left(\Lambda_{(1,2)} \widetilde{\nabla} d \phi\right)(X, Y, Z)=\frac{1}{2}\left(\widetilde{\nabla}_{Z} d \phi\right)(X, Y)+\begin{gathered}
\text { terms involving contractions and } \\
\text { symmetrisations of } d \phi \text { and } T^{*}
\end{gathered}
$$

Hence, we obtain along $\mathcal{Q}$ for $Z \in T \mathcal{Q}$ and $X,\left.Y \in T \widetilde{M}\right|_{\mathcal{Q}}$ that $\left(\widetilde{\nabla}_{Z} d \phi\right)(X, Y)=0$. By (13), (14), and (11) we get:

$$
h(\widetilde{\mathcal{R}}(X, Y) F, Z)=0,
$$

which is (b) of the second point.

Proposition 4.5. Let $\left(\mathcal{T}, h^{\mathcal{T}}, \nabla^{\mathcal{T}}\right)$ defined as above. Suppose that $\left.d \phi\right|_{\mathcal{Q}}=0,\left.T^{*}\right|_{\mathcal{Q}}=0$, $F\lrcorner T=T^{*}(., ., F)=0$, and that $\widetilde{R i c}$ denotes the Ricci curvature of the ambient connection $\widetilde{\nabla}$. Then $\nabla^{\mathcal{T}}$ is the normal standard tractor connection if and only if $\iota^{*} \widetilde{R i c}=0$.

Proof. First we note that equation (10) immediately implies that

$$
\widetilde{\mathcal{R}^{\mathcal{T}}(X, Y) Z}=\widetilde{\mathcal{R}}(\widetilde{X}, \widetilde{Y}) \widetilde{Z}
$$

where $\widetilde{\mathcal{R}}$ is the ambient curvature and the other tildes denote the corresponding identifications of Lemma 4.1. This implies on the one hand that for every $q \in Q$ the fibre $\mathcal{T}_{[q]}^{1}=\mathbb{R} \cdot\left[F_{q}\right] \subset \mathcal{T}_{[q]}$ is annihilated by $\mathcal{R}^{\mathcal{T}}(X, Y)$ for $X, Y \in T_{[q]} M$. This is one condition for the tractor connection to be normal.

In order to analyse the Ricci condition we fix a basis along $\mathcal{Q}$ of the form $\left(F, S_{1}, \ldots, S_{n}, Z\right)$ with $S_{i} \in \Gamma(T \mathcal{Q})$ such that $h\left(S_{i}, S_{j}\right)=\delta_{i j}$ and $Z \in \Gamma\left(\left.T \widetilde{M}\right|_{\mathcal{Q}}\right)$ such that $h(Z, F)=1$ and $h\left(Z, S_{i}\right)=0$. The Ricci curvature for $X, Y \in T \mathcal{Q}$ then is given by

$$
\widetilde{\operatorname{Ric}}(X, Y)=\underbrace{h(\widetilde{\mathcal{R}}(Z, X) Y, F)}_{=-h(\widetilde{\mathcal{R}}(Z, X) F, Y)=0}+\underbrace{h(\widetilde{\mathcal{R}}(F, X) Y, Z)}_{=h(\widetilde{\mathcal{R}}(Y, Z) F, X)=0}+\sum_{i=1}^{n} h\left(\widetilde{\mathcal{R}}\left(S_{i}, X\right) Y, S_{i}\right)
$$

by the previous lemma. Using the identification (15), the vanishing of the third summand is equivalent to the vanishing of the Ricci contraction of the tractor curvature on $\left(\mathcal{T}^{1}\right)^{\perp} / \mathcal{T}^{1} \simeq$ $T M[-1]$. This gives the statement.

\section{An ambient connection with Cotton-York tensor as torsion}

In this section we want to give an example of an ambient connection which realises the normal conformal Tractor holonomy.

We set $\widetilde{M}=\mathbb{R} \times M \times \mathbb{R}^{+}$. Let $s$ and $q$ be the coordinates along $\mathbb{R}$ and $\mathbb{R}^{+}$, with coordinate vector fields $S$ and $Q$, respectively. Throughout, we assume that we have fixed a metric $g$ in the conformal class and with it the corresponding Levi-Civita connection $\nabla$. The embedding of the bundle $\mathcal{Q}$ is given by

$$
\begin{aligned}
\iota: \quad \mathcal{Q} & \rightarrow \widetilde{M} \\
t^{2} g_{x} & \mapsto(0, x, t),
\end{aligned}
$$


i.e. $\mathcal{Q}=\{s=0\}$. This is equivariant w.r.t. the $\mathbb{R}^{+}$-action on $\mathcal{Q}$ given by $\varphi_{t}\left(g_{x}\right)=t^{2} g_{x}$ and on $\widetilde{M}$ given by

$$
\varphi_{t}(s, x, q)=(t s, x, t q)
$$

The fundamental vector field for this action is given by $F(s, x, q)=s S+q Q$.

In order to define the ambient metric we introduce the following endomorphisms. Denote by $\mathrm{P}$ the Schouten tensor of the fixed connection $\nabla$, considered (via $g$ ) as an endomorphism of $T M$. Using the identification of $T_{(s, x, q)} \widetilde{M}=\mathbb{R} S \oplus T_{x} M \oplus \mathbb{R} Q$ we define a bundle automorphism $f$ of $T \widetilde{M}$ by:

$$
\begin{aligned}
f(S) & =S \\
f(Q) & =Q \\
f(X) & =(s \mathrm{P}+q I d)^{-1} X
\end{aligned}
$$

for $X \in T_{x} M \subset T_{(s, x, q)} \widetilde{M}$. Of course, $f$ is not defined when $-s / q$ is the inverse of an eigenvalue of $\mathrm{P}$. However on $\{0\} \times M \times\{1\},-s / q=0$, so $f$ is defined for for small $s$. Notice that $\left.f\right|_{T M}$ commutes with $\mathrm{P}$ since $f^{-1}$ does.

For $X \in T_{x} M$ we can understand $f(X)$ as a lift to $\Gamma\left(\left.T \widetilde{M}\right|_{\mathbb{R}^{+} \cdot x}\right)$, which we denote by $\widetilde{X}$. A direct calculation shows that $\widetilde{X}$ is homogeneous of degree -1 , i.e. $\widetilde{X} \in \Gamma^{-1}\left(\left.T \widetilde{M}\right|_{\mathbb{R}^{+} \cdot x}\right)$, or, if $X \in \Gamma(T M)$, then $\widetilde{X} \in \Gamma^{-1}(T \widetilde{M})$ (c.f. Lemma 4.1). We use this lift to define a metric:

$$
\begin{aligned}
h(S, Q) & =1 \\
h(\widetilde{X}, \widetilde{Y}) & =g(X, Y), \text { for } X, Y \in T M, \text { and } 0 \text { otherwise. }
\end{aligned}
$$

This metric is by definition homogeneous of degree 2, and it also satisfies the second condition posed on an ambient metric: $\left(\iota^{*} h\right)_{q^{2} g_{x}}(X, Y)=h_{(0, x, q)}(X, Y)=q^{2} g(X, Y)$. Hence, the metric $h$ defines an ambient metric on $\widetilde{M}$. The form dual to $F$ is given by $\phi=s d q+q d s$, hence $d \phi=0$ everywhere.

Using the defined lifts we now want to define an ambient connection on $\widetilde{M}$ by:

$$
\begin{aligned}
\widetilde{\nabla}_{X} \widetilde{Y} & =\widetilde{\nabla_{X} Y}-g(X, Y) S-\mathrm{P}(X, Y) Q, \\
\widetilde{\nabla}_{X} Q & =\widetilde{X}, \\
\widetilde{\nabla}_{X} S & =\widetilde{\mathrm{P}(X)}, \\
\widetilde{\nabla}_{Q} X & =\widetilde{X} \\
\widetilde{\nabla}_{S} X & =\mathrm{P}(\widetilde{X})=\widetilde{P(X)},
\end{aligned}
$$

and all the other $Q$ and $S$ terms being zero.

Lemma 5.1. $\widetilde{\nabla} h=0$.

Proof. In the following we assume $X, Y, Z \in \Gamma(T M)$ and $U, V \in\{Q, S\}$. The first thing to notice is that $\left(\widetilde{\nabla}_{U} h\right)(V, X)=\left(\widetilde{\nabla}_{X} h\right)(U, V)=0$. Secondly we get that

$$
\begin{aligned}
\left(\widetilde{\nabla}_{X} h\right)(Q, \tilde{Y}) & =X(h(Q, \tilde{Y})-h(\widetilde{X}, \widetilde{Y})+g(X, Y) h(Q, S)=0, \text { and } \\
\left(\widetilde{\nabla}_{X} h\right)(S, \widetilde{Y}) & =X(h(S, \widetilde{Y})-h(\widetilde{P(X)}, \widetilde{Y})+P(X, Y) h(S, Q)=0 .
\end{aligned}
$$


Furthermore we have that

$$
\begin{aligned}
& (\widetilde{\nabla} Z h)(\tilde{X}, \tilde{Y})=Z(g(X, Y))-h\left(\widetilde{\widetilde{\nabla_{Z} X}}, \widetilde{Y}\right)-h\left(\widetilde{X}, \widetilde{\widetilde{\nabla}_{U} Y}\right) \\
& +g(Z, X) h(S, \tilde{Y})+g(Z, Y) h(S, \widetilde{X})+\mathrm{P}(Z, X) h(Q, \widetilde{Y})+\mathrm{P}(Z, Y) h(Q, \widetilde{X}) \\
& =\left(\widetilde{\nabla}_{Z} g\right)(X, Y) \\
& =0 \text {. }
\end{aligned}
$$

Finally one notices that the lifts $\widetilde{X}$ are $Q$ and $S$ parallel:

$$
\begin{aligned}
\widetilde{\nabla}_{Q} \widetilde{X} & =\left(\widetilde{\nabla}_{Q} f\right)(X)+f \circ f(X) \\
& =-I d \circ(s \mathrm{P}+q I d)^{-2}(X)+(s \mathrm{P}+q I d)^{-2}(X) \\
& =0, \\
\widetilde{\nabla}_{S} \widetilde{X} & \left.=\left(\widetilde{\nabla}_{S} f\right)(X)+f \circ P \circ f(X)\right) \\
& =-\mathrm{P} \circ(s \mathrm{P}+q I d)^{-2}(X)+\mathrm{P} \circ(s \mathrm{P}+q I d)^{-2}(X) \\
& =0 .
\end{aligned}
$$

This implies that $\left(\widetilde{\nabla}_{U} h\right)(\widetilde{X}, \widetilde{Y})=U(g(X, Y))-h\left(\widetilde{\nabla}_{U} \widetilde{X}, \widetilde{Y}\right)-h\left(\widetilde{X}, \widetilde{\nabla}_{U} \widetilde{Y}\right)=0$.

Lemma 5.2. The torsion $T$ of $\widetilde{\nabla}$ is given by the Cotton-York tensor of $g$, i.e.

$$
T(X, Y)=s \widetilde{C Y(X, Y)} \text { and } T^{*}(X, Y, \widetilde{Z})=s C Y(X, Y, Z)
$$

for $X, Y, Z \in T M$, and zero otherwise, where $C Y(X, Y)=\left(\nabla_{X} \mathrm{P}\right)(Y)-\left(\nabla_{Y} \mathrm{P}\right)(X)$ is the Cotton-York endomorphism, respectively its dualisation. In particular, $\left(\mathcal{L}_{F} T^{*}\right)=2 T^{*},\left.T\right|_{\mathcal{Q}}=$ 0 and $F\lrcorner T=T^{*}(., ., F)=0$.

Proof. For $X, Y \in T M$ we calculate:

$$
\begin{aligned}
T(S, Q) & =0 \\
T(X, Q) & =0 \\
T(X, S) & =\widetilde{\mathrm{P}(X)}-\mathrm{P}(\widetilde{X})=0, \text { since } f \text { commutes with } \mathrm{P}
\end{aligned}
$$

which implies $F\lrcorner T=0$. In order to get the term $T(X, Y)$ for $X, Y \in T M$ we need to calculate $\widetilde{\nabla}_{X} Y$. It is

$$
\begin{aligned}
\widetilde{\nabla}_{X} Y & =\widetilde{\nabla}_{X} f^{-1} \widetilde{Y} \\
& =\widetilde{\nabla}_{X}(s \mathrm{P}(\tilde{Y})+q \widetilde{Y}) \\
& =s \widetilde{\nabla}_{X} \widetilde{\mathrm{P}(Y)}+q \widetilde{\nabla_{X}} \widetilde{Y} \\
& =s\left(\widetilde{\nabla_{X} \mathrm{P}(Y)}-\mathrm{P}(X, Y) S-\mathrm{P}(X, \mathrm{P}(Y)) Q\right)+q\left(\widetilde{\nabla_{X} Y}-g(X, Y) S-\mathrm{P}(X, Y) Q\right) .
\end{aligned}
$$

This implies

$$
\begin{aligned}
T(X, Y) & =s\left(\widetilde{\nabla_{X} \mathrm{P}(Y)}-\widehat{\nabla_{Y} \mathrm{P}(X)}\right)+q\left(\widetilde{\nabla_{X} Y}-\widehat{\nabla_{X} Y}\right)-[X, Y] \\
& \left.=s\left(\widetilde{\nabla_{X} \mathrm{P}(Y)}-\widetilde{\nabla_{Y} \mathrm{P}(X)}\right)+q \widetilde{[X, Y]}-f^{-1([X, Y]}\right) \\
& \left.=s\left(\widetilde{\nabla_{X} \mathrm{P}(Y)}-\widetilde{\nabla_{Y} \mathrm{P}(X)}\right)-s \mathrm{P}(\widetilde{[X, Y}]\right) \\
& =s \widetilde{C Y(X, Y)}
\end{aligned}
$$


This gives immediately the formula for $T^{*},\left.T\right|_{\mathcal{Q}}=0$ and $T^{*}(., ., F)=0$. Also homogeneity two is easily verified:

$$
\begin{aligned}
\left(\mathcal{L}_{F} T^{*}\right)(X, Y, \widetilde{Z})= & F(s C Y(X, Y, Z)) \\
& -s(C Y(\underbrace{[F, X]}_{=0}, Y, \widetilde{Z})+C Y(X, \underbrace{[F, Y]}_{=0}, \widetilde{Z})+C Y(X, Y, \underbrace{[F, \widetilde{Z}]}_{=-\widetilde{Z}})) \\
= & 2 s C Y(X, Y, \widetilde{Z}) .
\end{aligned}
$$

A straightforward calculation gives the curvature of $\widetilde{\nabla}$.

Lemma 5.3. Let $W$ be the Weyl tensor and $C Y$ the Cotton-York tensor of the metric $g$. For $X, Y, Z \in T M$ the curvature of $\widetilde{\nabla}$ is given by

$$
\widetilde{\mathcal{R}}(X, Y) \widetilde{Z}=W \widetilde{(X, Y)} Z-C Y(X, Y, Z) \cdot Q,
$$

and all other terms not determined by the symmetries of the curvature terms being zero. In particular, $\widetilde{\mathcal{R}}(U, V) F=0$ along $\mathcal{Q}$ for all $U,\left.V \in T \widetilde{M}\right|_{\mathcal{Q}}$.

By this we obtain the Ricci-curvature of $\widetilde{\nabla}$.

Lemma 5.4. The Ricci curvature of $\widetilde{\nabla}$ vanishes along $\mathcal{Q}$.

Proof. Let $\left\{E_{i}\right\}_{i=1}^{n}$ be a basis of $T_{x} M$ orthonormal w.r.t. $g$. Thus $\left(S, \widetilde{E}_{1}, \ldots, \widetilde{E}_{n}, Q\right)$ is a basis of $T_{(s, x, q)} \widetilde{M}$ in which the Ricci-curvature in $(s, x, q)$ can be written as

$$
\widetilde{\operatorname{Ric}}(U, V)=h(\widetilde{\mathcal{R}}(S, U) V, Q)+h(\widetilde{\mathcal{R}}(Q, U) V, S)+\sum_{i=1}^{n} h\left(\widetilde{\mathcal{R}}\left(\widetilde{E}_{i}, U\right) V, \widetilde{E}_{i}\right) .
$$

This implies $\widetilde{\operatorname{Ric}}(Q,)=$.0 and $\widetilde{\operatorname{Ric}}(S, S)=0$. Furthermore we obtain

$$
\begin{aligned}
& \widetilde{\operatorname{Ric}}(X, S)=\sum_{i=1}^{n} C Y\left(\widetilde{E}_{i}, X, E_{i}\right) \text { and } \\
& \widetilde{\operatorname{Ric}}(X, \widetilde{Y})=\sum_{i=1}^{n} g\left(W\left(\widetilde{E}_{i}, X\right) Y, E_{i}\right) .
\end{aligned}
$$

But along $\mathcal{Q}$ we have that $\widetilde{E}_{i}=\frac{1}{q} E_{i}$ and thus $\widetilde{\operatorname{Ric}}=0$ along $\mathcal{Q}$

We can summarize:

Theorem 5.5. $(\widetilde{M}, h, \widetilde{\nabla})$ is an ambient manifold with ambient metric $h$ and ambient connection $\widetilde{\nabla}$ with torsion $T$, satisfying $d \phi=0,\left.T\right|_{\mathcal{Q}}=0$ and $\left.F\right\lrcorner T=T^{*}(., ., F)=0$. The corresponding conformal standard Tractor connection is normal.

But we can show even more.

Proposition 5.6. The holonomy of $\widetilde{M}$ is generated by paths in the embedded manifold $\{0\} \times$ $M \times\{1\}$, i.e. $\operatorname{Hol}_{(0, x, 1)}(T \widetilde{M}, \widetilde{\nabla})=\operatorname{Hol}_{(0, x, 1)}\left(\left.T \widetilde{M}\right|_{M}, \widetilde{\nabla}\right)$. 
Proof. Assume now that $\phi:[0,1] \rightarrow\{0\} \times M \times\{1\}$ is a path with tangent field $Y$. We parallel transport any given vector of $T N$ along $\phi$, getting the equation:

$$
\begin{aligned}
0 & =\widetilde{\nabla}_{Y}(X+a Q+b S) \\
& =\left(\nabla_{Y} X+a Y+b \mathrm{P}(Y)\right)-g(Y, X) S-\mathrm{P}(Y, X) Q+Y(a) Q+Y(b) S .
\end{aligned}
$$

for some section $X$ of $\left.T M\right|_{\phi}, a$ and $b$ functions on $M$. The lift of this section into the whole of $\widetilde{M}$ is $\widetilde{X}+a Q+b S$. To prove that the holonomy transform is the same along any lift of $\phi$, it suffices to prove

$$
\begin{aligned}
& \widetilde{\nabla}_{Y}(\tilde{X}+a Q+b S)=0 \\
& \widetilde{\nabla}_{Q}(\widetilde{X}+a Q+b S)=0 \\
& \widetilde{\nabla}_{S}(\widetilde{X}+a Q+b S)=0 .
\end{aligned}
$$

However this is the case, since

$$
\begin{aligned}
\widetilde{\nabla}_{Y}(\tilde{X}+a Q+b S)= & f\left(\nabla_{X} Y+a Y+b \mathrm{P}(Y)\right) \\
& -g(X, Y) S-\mathrm{P}(X, Y) Q+Y(a) Q+Y(b) S \\
= & 0,
\end{aligned}
$$

and the other two equalities were proved in Lemma 5.1

Theorem 5.7. The affine holonomy of $\widetilde{\nabla}$ is the same as the Tractor holonomy of $\nabla^{\mathcal{T}}$.

Proof. We identify $T \widetilde{M}$ with $\mathcal{T}-\mathcal{T}$ being split as in (11) by the choice of $\nabla$ - via

$$
\left(\begin{array}{l}
1 \\
0 \\
0
\end{array}\right)=S, \quad\left(\begin{array}{c}
0 \\
X \\
0
\end{array}\right)=X, \quad\left(\begin{array}{l}
0 \\
0 \\
1
\end{array}\right)=Q .
$$

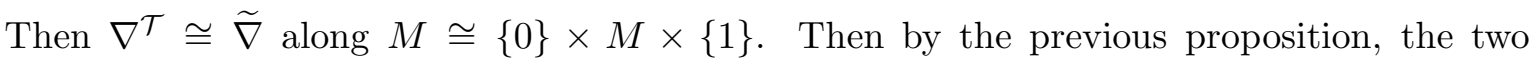
connections have same holonomy.

Theorem 5.8. If there is a metric in the conformal class with vanishing Cotton-York tensor, then $(\widetilde{M}, h)$ is an ambient metric in the sense of [CّG03]. In this case, the normal conformal tractor holonomy is the holonomy of the Levi-Civita connection of the semi-Riemannian manifold $(\widetilde{M}, h)$.

If there is a metric in the conformal class which is Einstein, $(\widetilde{M}, h)$ gives a Ricci-flat ambient metric in the sense of [FG85]. In particular, it gives the cone construction in the conformal Einstein case with $S \neq 0$ (see [Leit04] and [Arm05]), and the degenerate cone in the Ricci-flat case (see [Lei05]).

Proof. If $C Y=0$ the connection is torsion free and thus the Levi-Civita connection of $h$. In the Einstein case, it suffices to restrict attention to the sub-manifold

$$
\mathbb{R}^{+}\left(\left\{-\mu^{-1}\right\} \times M \times\{1\}\right),
$$

when $S+\mu Q$ is the preserved vector, to get the Einstein cone construction. In the Ricci-flat case, $f=\frac{1}{q} I d$, and thus $h=2 d s d q+q^{2} g$ which is the degenerate cone construction. Note that in the latter case the ambient connection is singularity free. 
Remark 5.9. $\widetilde{M}$ is not conformally invariant: Notice that is $\mathrm{P} \neq 0$ at $x \in M$, it must have an eigenvalue $q \neq 0$ at $x$. Then the connection is singular along

$$
\mathbb{R}^{+}(\{1\} \times M \times\{-q\}) .
$$

This shows that a conformally Ricci-flat metric that is not Ricci-flat itself gives rise to a connection with singularities. These singularities are not removable, as the term

$$
\widetilde{\nabla}_{Q} X=\widetilde{X}
$$

demonstrates. Thus $\widetilde{\nabla}$ is not a conformally invariant construction (even though it has the same holonomy as the conformally invariant Tractor connection).

Remark 5.10. In [Lei05] we proved algebraically that the normal Tractor holonomy algebra of a conformal class which contains a metric with zero Cotton-York tensor is a Berger algebra. The present approach gives a geometric demonstration of this proven fact because the constructed ambient connection has no torsion if we start the construction with a metric with zero Cotton York tensor. This implies that its curvature satisfies the first Bianchi-identity, and hence, its holonomy algebra is a Berger algebra.

Remark 5.11. Alternatively, we may define an affine connection on $\widetilde{M}$ rather crudely by:

$$
\begin{aligned}
\widetilde{\nabla}_{X} Y & =\nabla_{X} Y-q g(X, Y) S-q \mathrm{P}(X, Y) Q, \\
\widetilde{\nabla}_{X} Q & =\frac{1}{q} X, \\
\widetilde{\nabla}_{X} S & =\frac{1}{q} \mathrm{P}(X), \\
\widetilde{\nabla}_{Q} X & =\frac{1}{q} X, \\
\widetilde{\nabla}_{S} X & =0,
\end{aligned}
$$

and all the other $S$ and $Q$ terms being zero. Although this gives no ambient connection with the properties required in the previous sections, a direct calculation establishes that $\widetilde{\nabla}$ also has same holonomy as the tractor connection. Moreover its torsion only involves terms with $S$ and a tangent vector to $M$ - unlike the connection of the previous example, which generically has torsion between two vector fields of $M$ - and has no singularities.

\section{Tractor holonomy and the ambient connection}

In this last section, we aim to give some general holonomy related results, which might help in the construction of ambient connections whose holonomy is the same as the Tractor holonomy. The ideal would be to thus generate either a conformally invariant construction, or one with a torsion that allows one to put strong conditions on the holonomy.

As always, let $\widetilde{M}$ be an ambient manifold with ambient metric $h$ and ambient connection

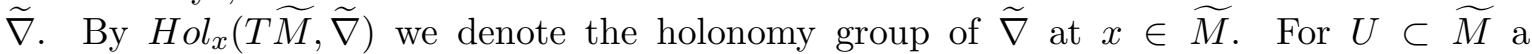
submanifold and $x \in U$ we denote by $\operatorname{Hol}_{x}\left(\left.T \widetilde{M}\right|_{U}, \widetilde{M}\right)$ the group generated by loops running in $U$ around $x$. 
Lemma 6.1. Let $\widetilde{M}$ be an ambient manifold with ambient metric $h$ and ambient connection $\widetilde{\nabla}$ such that $\left.d \phi\right|_{\mathcal{Q}}=\left.T\right|_{\mathcal{Q}}=0$. Assume furthermore that the flow of the $\mathbb{R}^{+}$-action is geodesic w.r.t. $\widetilde{\nabla}$, i.e. $\varphi_{t}(q)$ is a geodesic for each $q \in \mathcal{Q}$. Suppose we are given a global section $\sigma \in \Gamma\left(\mathcal{Q} \rightarrow M=\mathcal{Q} / \mathbb{R}^{+}\right)$. Then for any $q \in \sigma(M)$ it holds

$$
\operatorname{Hol}_{q}\left(\left.T \widetilde{M}\right|_{\mathcal{Q}}, \widetilde{\nabla}\right)=\operatorname{Hol}_{q}\left(\left.T \widetilde{M}\right|_{\sigma(M)}, \widetilde{\nabla}\right)
$$

Proof. First we want to collect some consequences of the assumption that the action of $\mathbb{R}^{+}$ defines geodesics. The tangent vector field to a geodesic $\varphi_{t}(q)$ for $q \in \sigma(M) \subset \mathcal{Q}$, denoted by $\Phi$ is related to the fundamental vector field $F$ as follows

$$
\begin{aligned}
F\left(\varphi_{t}(q)\right) & =\frac{d}{d s}\left(\varphi_{e^{s}} \circ \varphi_{t}(q)\right)_{\mid s=0}=\frac{d}{d s}\left(\varphi_{t e^{s}}(q)\right)_{\mid s=0}=t \cdot \frac{d}{d s}\left(\varphi_{s}(q)\right)_{\mid s=t} \\
& =t \cdot \Phi\left(\varphi_{t}(q)\right)=t \cdot \Phi(t)
\end{aligned}
$$

Since $\widetilde{\nabla}_{\Phi(t)} \Phi(t)=0$ this implies that

$$
\left(\widetilde{\nabla}_{F} F\right)\left(\varphi_{t}(q)\right)=\widetilde{\nabla}_{F}(t \Phi)=F(t) \Phi\left(\varphi_{t}(q)\right)+t \underbrace{\widetilde{\nabla}_{F} \Phi}_{=0}=t \Phi(\varphi(t))=F\left(\varphi_{t}(q)\right)
$$

Furthermore, if $X \in \Gamma\left(\left.T \mathcal{Q}\right|_{\sigma(M)}\right)$ we denote by $\widetilde{X}$ the lifted vector field given by

$$
\widetilde{X}\left(\varphi_{t}(q)\right)=\left(d \varphi_{t}\right)_{q}(X(q))
$$

Obviously it is $\widetilde{F}(\varphi(q))=F\left(\varphi_{t}(q)\right)$. More importantly, the vector fields $\widetilde{X}$ are homogeneous of degree 0 . Since the torsion and $d \phi$ vanishes on $\mathcal{Q}$ (8) implies that

$$
0=[F, \widetilde{X}]=\widetilde{\nabla}_{F} \widetilde{X}-\widetilde{\nabla}_{\widetilde{X}} F=\widetilde{\nabla}_{F} \widetilde{X}-\widetilde{X}
$$

Since $\varphi_{t}(q)$ are geodesics it is

$$
\widetilde{\nabla}_{\widetilde{X}} \tilde{Y}=\left(d \varphi_{t}\right)\left(\widetilde{\nabla}_{X} Y\right)=\widetilde{\widetilde{\nabla}_{X} Y}
$$

Now we take a curve $\widetilde{\gamma}$ in $\mathcal{Q}$. Then $\widetilde{\gamma}$ is given by $\widetilde{\gamma}(t)=\varphi_{f(t)}(\gamma(t))$ for $f$ a parameter transformation and $\gamma$ a curve in $\sigma(M)$. Its tangent vector is

$$
\begin{aligned}
\dot{\tilde{\gamma}}(t) & =\dot{f}(t) \Phi(\widetilde{\gamma}(t))+d \varphi_{f(t)}(\dot{\gamma}(t)) \\
& =\frac{\dot{f}(t)}{f(t)} F(\widetilde{\gamma}(t))+\widetilde{\dot{\gamma}(t)} \\
& =\frac{\dot{f}(t)}{f(t)} \widetilde{F}(\gamma(t))+\widetilde{\dot{\gamma}(t)}
\end{aligned}
$$

For the curve $\gamma$ in $\sigma(M)$ now we consider the vector field $Y$ which is assumed to be parallel displaced along $\gamma$. $Y$ can be written as

$$
Y(t)=a(t) F(\gamma(t))+X(t)
$$

with $a: \mathbb{R} \rightarrow \mathbb{R}$ and $X$ a vector field of $T \sigma(M)$ along $\gamma$. Hence we have

$$
\begin{aligned}
0=\widetilde{\nabla}_{\dot{\gamma}(t)} Y(t) & =\dot{a}(t) F(\gamma(t))+a(t) \widetilde{\nabla}_{\dot{\gamma}(t)} F+\widetilde{\nabla}_{\dot{\gamma}(t)} X(t) \\
& =\dot{a}(t) F(\gamma(t))+a(t) \dot{\gamma}(t)+\widetilde{\nabla}_{\dot{\gamma}(t)} X(t)
\end{aligned}
$$


Now consider the vector field $U(t)$ along $\widetilde{\gamma}(t)$ given by

$$
\begin{aligned}
U(t) & =a(t) \Phi(\widetilde{\gamma}(t))+\frac{1}{f(t)} d \varphi_{f(t)}(X(t)) \\
& =\frac{a(t)}{f(t)} F(\widetilde{\gamma}(t))+\frac{1}{f(t)} \widetilde{X(t)} .
\end{aligned}
$$

This vector field is parallel along $\widetilde{\gamma}$, because

$$
\begin{aligned}
\widetilde{\nabla}_{\dot{\tilde{\gamma}}(t)} U(t)= & \left(\frac{\dot{a}(t)}{f(t)}-a(t) \frac{\dot{f}(t)}{f^{2}(t)}\right) F(\widetilde{\gamma}(t))-\frac{\dot{f}(t)}{f^{2}(t)} \widetilde{X(t)} \\
& +\frac{a(t)}{f(t)}(\frac{\dot{f}(t)}{f(t)} \underbrace{\left(\widetilde{\nabla}_{F} F\right)}_{=F}(\widetilde{\gamma(t)}+\underbrace{\widetilde{\tilde{\gamma}(t)} F}_{=\widetilde{\dot{\gamma}}(t)})+\frac{1}{f(t)}(\frac{\dot{f}(t)}{f(t)} \underbrace{\widetilde{X(t)}}_{=\widetilde{\nabla_{F}} \widetilde{X(t)}}+\widetilde{\nabla}_{\tilde{\dot{\gamma}}(t)} \widetilde{X(t)}) \\
= & \frac{1}{f(t)}\left(\dot{a}(t) F(\widetilde{\gamma}(t))+a(t) \widetilde{\dot{\gamma}(t)}+\widetilde{\widetilde{\nabla}_{\tilde{\gamma}(t)}} \widetilde{X(t)}\right) \\
= & \frac{1}{f(t)}\left(\dot{a}(t) F(\gamma(t))+\widetilde{a(t) \dot{\gamma}(t)}+\widetilde{\nabla}_{\dot{\gamma}(t)} X(t)\right) \\
= & 0 .
\end{aligned}
$$

But for a loop $\widetilde{\gamma}$ around $q \in \sigma(M)$, i.e. $\widetilde{\gamma}(0)=\widetilde{\gamma}(1)=q$ we have that $f(0)=f(1)=1$ and $\gamma(0)=\gamma(1)=q$ is also a loop. But this implies that $Y(1)=U(1)$ and hence the holonomies are the same.

This lemma immediately implies:

Proposition 6.2. Let $\widetilde{M}$ be an ambient manifold with ambient metric $h$ and ambient connection $\widetilde{\nabla}$ such that $\left.d \phi\right|_{\mathcal{Q}}=T_{\mathcal{Q}}=0$, i.e. $(\widetilde{M}, h, \widetilde{\nabla})$ defines a standard conformal tractor bundle. If the flow of the $\mathbb{R}^{+}$-action is geodesic w.r.t. $\widetilde{\nabla}$, then the conformal tractor holonomy $\operatorname{Hol}_{[q]}\left(\mathcal{T}, \nabla^{\mathcal{T}}\right)$ is isomorphic to $\operatorname{Hol}_{q}\left(\left.T \widetilde{M}\right|_{\mathcal{Q}}, \widetilde{\nabla}\right)$.

Getting these groups to be equal to the full $\operatorname{Hol}_{q}(T \widetilde{M}, \widetilde{\nabla})$ is more tricky, however, and requires extra considerations beyond the scope of this paper.

\section{References}

[Arm05] Stuart Armstrong. Conformal Holonomy: A Classification, 2005. arXiv:math.DG/0503388.

[BEG94] T. N. Bailey, M. G. Eastwood, and A. R. Gover. Thomas's structure bundle for conformal, projective and related structures. Rocky Mountain J. Math., 24(4):11911217, 1994.

[ČG02] Andreas Čap and A. Rod Gover. Tractor calculi for parabolic geometries. Trans. Amer. Math. Soc., 354(4):1511-1548 (electronic), 2002.

[ČG03] Andreas Čap and A. Rod Gover. Standard tractors and the conformal ambient metric construction. Ann. Global Anal. Geom., 24(3):231-259, 2003. 
[Car23] E. Cartan: Les Espaces à Connexion Conform, Les Annales de la Société Polonaise de Mathématiques, 2, 171-202, 1923.

[Eas96] Michael Eastwood. Notes on conformal differential geometry. In The Proceedings of the 15th Winter School "Geometry and Physics" (Srni, 1995), number 43 in Supplemento ai Rendiconti del Circolo Matematico di Palermo. Serie II, pages 5776, 1996.

[FG85] Charles Fefferman and C. Robin Graham. Conformal invariants. Astérisque, (Numero Hors Serie):95-116, 1985. The mathematical heritage of Élie Cartan (Lyon, 1984).

[FG02] Charles Fefferman and C. Robin Graham. Q-curvature and Poincaré metrics. Math. Res. Lett., 9(2-3):139-151, 2002.

[FH03] Charles Fefferman and Kengo Hirachi. Ambient metric construction of $Q$-curvature in conformal and CR geometries. Math. Res. Lett., 10(5-6):819-831, 2003.

[Leit04] Felipe Leitner. Normal Conformal Killing Forms, 2004. math.DG/0406316 at http://arxiv.org.

[Lei05] Thomas Leistner. Conformal holonomy of C-spaces, Ricci-flat, and Lorentzian manifolds, 2005. math.DG/0501239 at http://arxiv.org, Differential Geometry and its Applications, in press.

[Tho26] Tracy Yerkes Thomas. On conformal geometry. Proc. Nat. Acad. Sci., 12:352-359, 1926.

[Tho32] Tracy Yerkes Thomas. Conformal tensors. Proc. Nat. Acad. Sci., 18:103-189, 1932. 